\title{
TOPICS IN DIAGNOSTIC IMAGING
}

\section{Retropharyngeal Calcific Tendonitis in a Patient Seeking Chiropractic Care for Neck Pain: A Case Report}

\author{
Laura Munson, DC, Matthew F. Funk, DC, and Terence A. Perrault, DC, DABCR
}

\begin{abstract}
Objective: The purpose of this report is to describe the evaluation and treatment of a patient initially presenting for chiropractic care of neck pain (NP) who was subsequently diagnosed with retropharyngeal calcific tendonitis (RCT). Clinical Features: A 53-year-old woman presented to a chiropractic office with severe idiopathic NP, loss of cervical mobility, and intermittent painful swallowing. She had a motor vehicle accident 6 months before with NP that resolved within a week without treatment. A brief examination and some manual palliative care were attempted by the chiropractor. Two days later, the patient developed severe NP and fever, sought emergency medical care on her own, and requested the chiropractor meet her at the hospital. The emergency physicians initially considered an infection, and antibiotics were provided along with oxycodone and naproxen sodium for pain. After advanced (computed tomography) imaging, RCT was diagnosed, and she was discharged the next day.

Interventions and Outcome: After being diagnosed, the patient's primary care provider prescribed prednisone and physical therapy. After 3 weeks, the patient stated her pain continued and she went for chiropractic care and was treated for mechanical neck pain with manipulation, manual muscle treatment, postural training, and rehabilitative exercises.

Conclusion: Practitioners should recognize the clinical presentation of RCT, order appropriate computed tomography imaging, and implement effective treatments. Although uncommon, RCT should be considered in patients with NP, limited mobility, and painful swallowing. (J Chiropr Med 2018;17:275-282)

Key Indexing Terms: Chiropractic; Neck Pain; Tendinopathy
\end{abstract}

\section{INTRODUCTION}

It is estimated that less than $0.4 \%$ of acute neck pain (NP) cases are due to serious conditions. ${ }^{1}$ Murphy states severe pathology can be expected in about $1 \%$ to $3 \%$ of all patients with cervical disorders. ${ }^{2}$ Retropharyngeal calcific tendonitis (RCT) is an uncommon and potentially misdiagnosed cause of acute non-traumatic NP. ${ }^{3,4}$ Retropharyngeal calcific tendonitis, also known as prevertebral calcific tendonitis and longus colli hydroxyapatite deposition disease, is often associated with acute debilitating NP. Retropharyngeal calcific tendonitis is typically

University of Bridgeport College of Chiropractic, Bridgeport, Connecticut.

Corresponding author: Matthew F. Funk, DC, University of Bridgeport College of Chiropractic, 858 Freeman Ave, Stratford, CT 06614. Tel.: +1 8602276320 .

(e-mail: mfunk@bridgeport.edu).

Paper submitted December 1, 2017; in revised form March 19, 2018; accepted April 2, 2018.

1556-3707

(C) 2018 National University of Health Sciences.

https://doi.org/10.1016/j.jcm.2018.04.006 benign and self-limiting, but can be misdiagnosed as retropharyngeal abscess, delaying correct treatment. Airway obstruction, dysphagia, or severe idiopathic NP necessitate immediate medical attention. ${ }^{5}$

The purpose of this case report is to discuss the initial evaluation, medical treatment, and follow-up chiropractic treatment and rehabilitation of a patient with RCT, NP, and cervicothoracic mechanical dysfunction who presented for chiropractic care for NP.

\section{CASE RePORT \\ Initial Presentation}

A 53-year-old woman presented for chiropractic care with recent-onset NP and stiffness that came on over a few days and was worsened by lifting. Initial pain was reported as 8 of 10 on the numerical pain rating scale (NPRS). Swallowing was intermittently painful. She held her head in slight flexion, and her gait was guarded. All cervical ranges of motion were limited with pain, and she was unable to extend the neck without severe pain. Palpation revealed 
hypertonic and tender cervical musculature. Orthopedic provocative tests were not performed owing to severe pain. There were no palpable masses in her neck, and cervical lymph nodes were non-tender.

The patient had experienced episodic NP and lower back pain over the previous 15 years and periodically sought treatments including chiropractic care, physical therapy, and prescription medications, all providing relief to some degree. Six months before initial presentation, she was involved in a motor vehicle accident (MVA) in which the car she was driving hit a small car in the rear while travelling approximately 30 miles per hour. She experienced left hand and wrist pain as well as moderate NP that resolved within a week without treatment.

At the time of initial presentation the patient worked as a full-time nanny where she regularly lifted a 15-month-old toddler, performed housecleaning, and cared for 3 other children. She stated that she did no regular exercise. She was on medications for hypothyroidism, fibromyalgia, sleep apnea, narcolepsy, hypertension, and gastroesophageal reflux disease. Surgical history included hysterectomy, cholecystectomy, hernia repair, 3 cesarean sections, heel spur removal, and release of stenosing tenosynovitis in the left wrist. She was taking the following medications: levothyroxine, $112 \mathrm{mcg}$ daily; tramadol, $50 \mathrm{mg}, 2$ tablets twice per day; amlodipine-atorvastatin, 5 to $20 \mathrm{mg}$ daily; dexlansoprazole, $60 \mathrm{mg}$ daily; armodafinil, $250 \mathrm{mg}$ daily; hydrochlorothiazide, $12.5 \mathrm{mg}$ daily; orphenadrine, $100 \mathrm{mg}$ twice per day; and zolpidem, $10 \mathrm{mg}$ daily.

Owing to pain and limited motion, further evaluation was deferred. No radiographs were ordered at the time of the initial presentation, as the absence of immediate trauma and lack of red flags suggested a trial of conservative treatment. The patient and doctor of chiropractic agreed that imaging would be ordered if there was no progress within 24 hours. Gentle massage was given in an attempt to provide symptomatic relief.

\section{Emergency Department Examination and Medical Treatment}

Two days later, the patient developed increased NP and a fever of $103^{\circ} \mathrm{F}$. She went to the emergency department on her own and phoned the attending chiropractor, who met the patient there and observed the emergency department physicians in their effort to provide a diagnosis. The medical physicians obtained 2 plain film views of the chest, read as negative for active disease, and gave intravenous morphine that failed to provide relief. Dilaudid (hydromorphone) subsequently was given, which slightly alleviated pain. Blood tests showed an elevated white blood cell count of 14.8. Other abnormal lab tests results included low red blood cell count, low hemoglobin, and low hematocrit. Later at the hospital, the patient's temperature decreased from $103^{\circ} \mathrm{F}$ to $97^{\circ} \mathrm{F}$. Other vitals were normal.
While the patient was medicated, magnetic resonance imaging (MRI), computed tomography (CT), and CT with contrast were performed next. CT findings of retropharyngeal soft tissue thickening led to an initial suspicion of infection and abscess. Computed tomography with contrast also revealed retropharyngeal calcification within the longus colli tendon (Figs 1 and 2). The MRI was performed after $\mathrm{CT}$ and showed thickening of the longus colli tendons with prevertebral retropharyngeal fluid collection from the clivus to the lower cervical spine (Fig 3). The initial report also stated that differentials should include retropharyngeal abscess or, less likely, avulsion injury to the osseousligamentous junction of the anterior cervical spine. No evidence of osseous-ligamentous disruption or concomitant osseous reactive medullary edema or hemorrhage was seen on MRI, ruling out avulsion injury. In cases of retropharyngeal abscess, the CT with contrast would be expected to demonstrate ring or border enhancement surrounding the abscess, which was not present in this case. The radiologist and orthopedic surgeon conferred and decided on a diagnosis of RCT. Degenerative disc disease and mild spondylosis at C4-C5 and C5-C6 also were found in the imaging studies. There was a slight compromise of the spinal canal at the $\mathrm{C} 5$ and $\mathrm{C} 6$ levels, but no severe spinal stenosis was evident.

The patient was discharged from the hospital after staying overnight and was prescribed clindamycin (antibacterial), $300 \mathrm{mg} 3 \times$ a day for 5 days; oxycodone, $5 \mathrm{mg} 1$ to 2 tablets every 4 to 6 hours as needed; and naproxen sodium (Naprosyn), $250 \mathrm{mg}$ twice per day for 10 days along with her regular medications: Synthroid, tramadol, soma, and flexeril.

A few days after discharge from the hospital, there was a follow-up visit with her primary care physician, who prescribed a 10-day course of prednisone and discontinued naproxen sodium. Prednisone helped to decrease the pain quite a bit. She was referred to physical therapy where she received deep massage, traction, and hand bicycle exercises. After 3 weeks of therapy, the patient chose to discontinue because it aggravated her symptoms.

\section{Follow-up Chiropractic History, Examination, and Treatment}

History. Approximately 8 weeks after initial presentation, the patient returned to the chiropractic office with ongoing severe NP, stiffness especially when trying to look up, and daily intermittent painful swallowing. She still rated the neck pain 8 of 10 on the NPRS. Pain was provoked by movement such as grooming, household chores, lifting, or driving more than 1 hour. She stated nothing provided relief and she avoided provocative activities. There was no radiating pain, altered sensations, or weakness. Pain was reported throughout the day, but it did not interrupt sleep. She denied any other changes in her medical history and 


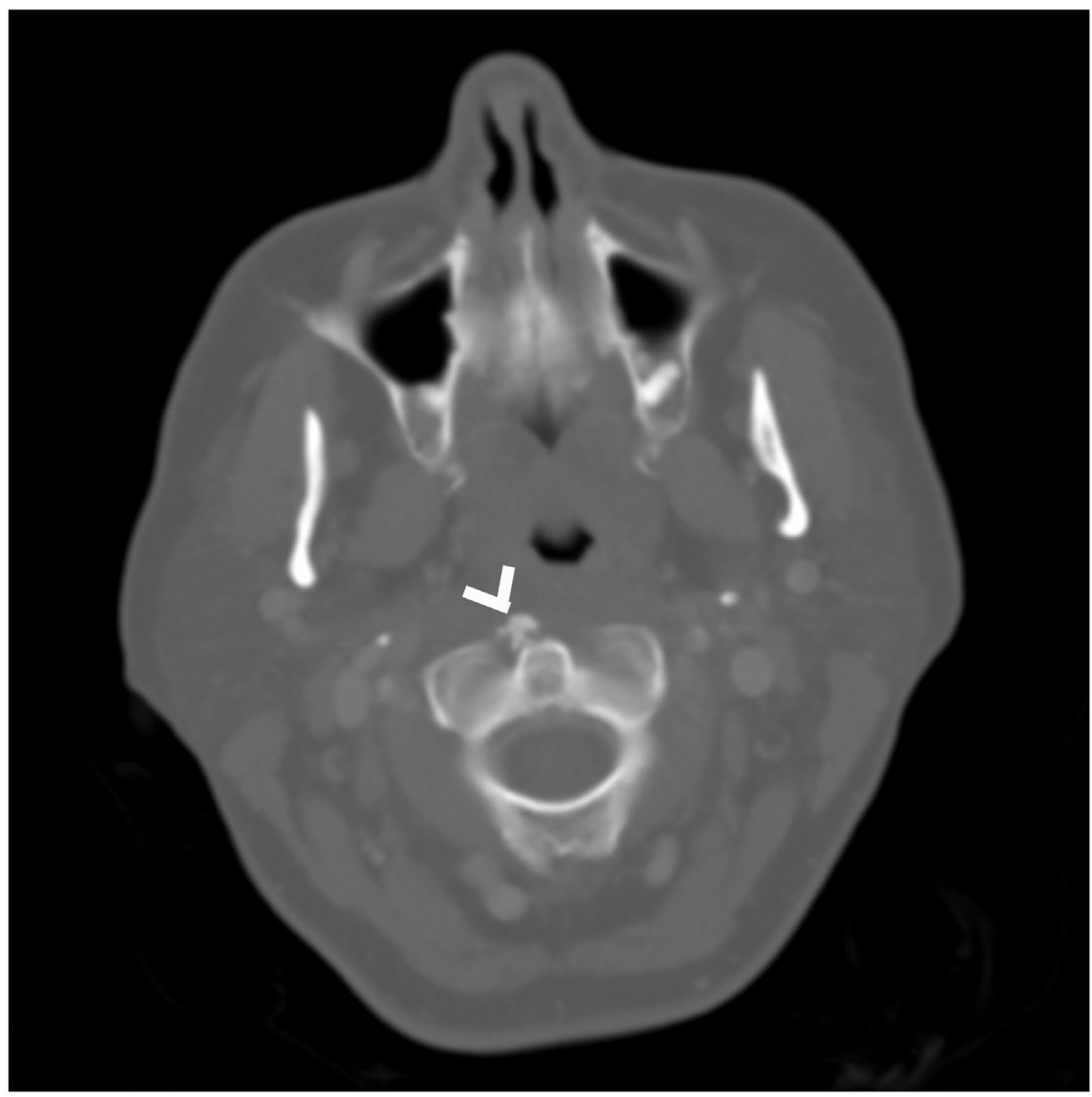

Fig I. Computed tomography scan (bone window) demonstrates a globular calcification (white arrow head) within the soft tissues anterior to the anterior tubercle of C1. This represents calcification within the tendinous structures of the longus colli.

continued with her regular medications since the first visit 8 weeks prior.

Examination. She was unable to lie supine without support in flexion. A postural exam revealed upper crossed syndrome with forward head posture, markedly elevated shoulders, forward rounded shoulders, and protracted scapulae. Dysfunctional breathing was noted as the patient elevated her shoulders during inspiration. Chest breathing was more predominant than abdominal breathing.

Cervical spine active ranges of motion were limited with increased pain at end range: extension $0^{\circ}$, flexion $40^{\circ}$, right and left rotation $40^{\circ}$, left lateral flexion $20^{\circ}$, and right lateral flexion $25^{\circ}$. Bilateral shoulder ranges of motion were full. Active left shoulder abduction at $70^{\circ}$ produced left anterior shoulder pain. Resisted left shoulder abduction and palpation over the left supraspinatus tendon were painful. Thoracic spine ranges were full and pain free except for limited extension $\left(20^{\circ}\right)$.

Palpation revealed moderate to severe hypertonicity and tenderness in the following muscles bilaterally: upper trapezius, levator scapula, scalenes, sternocleidomastoid, and pectoralis major and minor. The right serratus anterior was hypertonic compared to the left. Posterior to anterior springing on facets at $\mathrm{C} 2, \mathrm{C} 6-7, \mathrm{~T} 1-2$, and $\mathrm{T} 4$ provoked local pain.

Upper-extremity sensation, motor, and reflex tests were normal bilaterally. Axial cervical compression produced neck pain without radiation. Distraction provoked neck pain. An elevated arm stress test provoked numbness down both arms into the fourth and fifth digits. Adson's sign (diminished radial pulse in the fully extended arm when the head is rotated to the ipsilateral side with extended neck after deep inspiration) was present and provoked numbness in her right hand and all fingers.

An initial Neck Disability Index (NDI) was completed with a score of 30 , indicating severe disability. ${ }^{6}$ The pain diagram demonstrated well-delineated symbols, which suggested consistent symptom reporting. ${ }^{7}$ The patient completed the Yellow Flag Form, which may predict her risk for chronicity or disability, and scored 50 of 130 (low risk). ${ }^{8}$

Chiropractic Treatment. Diagnoses included RCT, cervicothoracic dysfunction, hypertonicity of cervicothoracic 


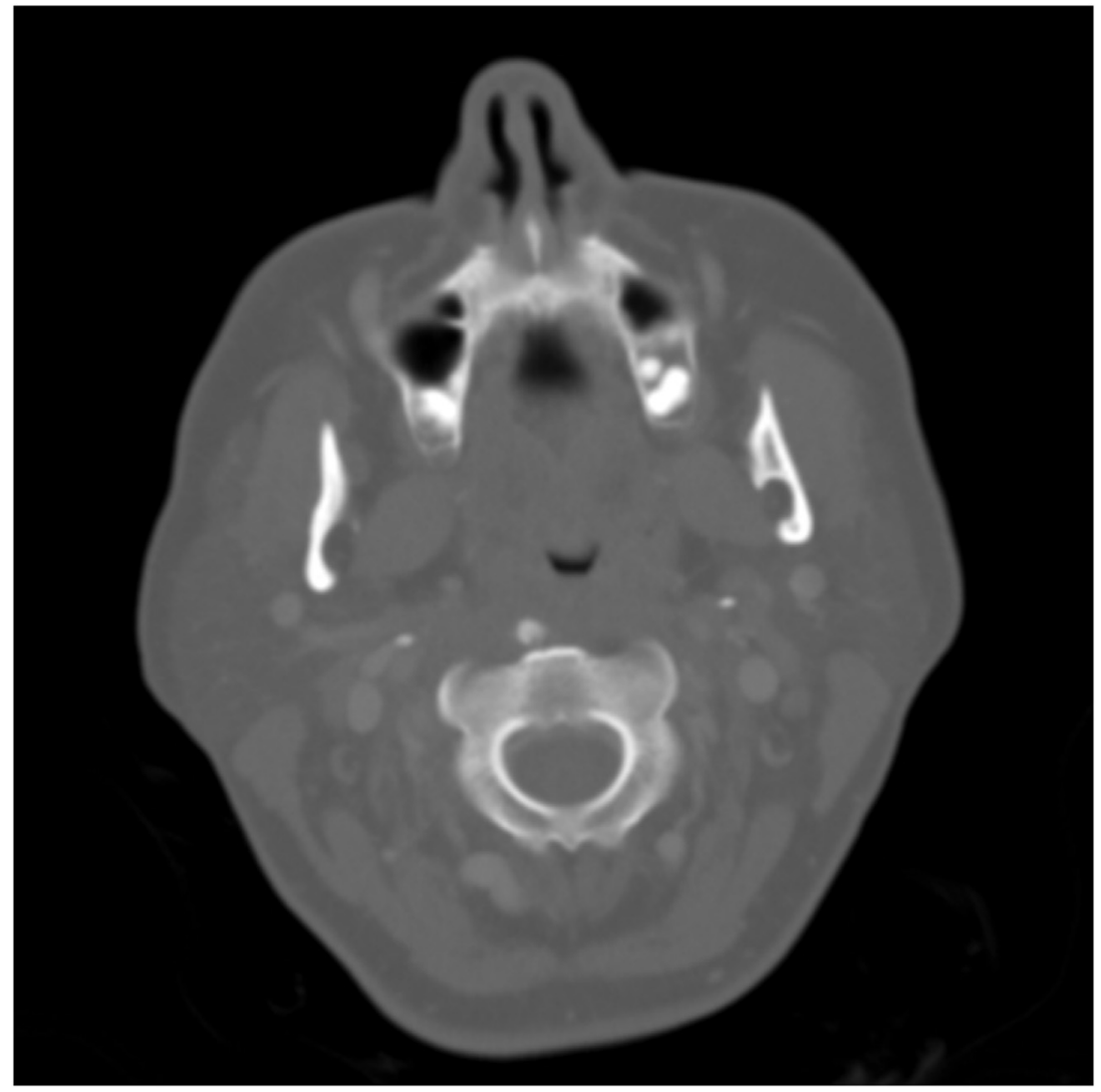

Fig 2. Computed tomography scan (bone window) a few millimeters below Figure 1 demonstrates a circular calcification within the C2 prevertebral soft tissues. This finding strongly suggests calcium infiltration within the longus colli tendon.

muscles, supraspinatus tendinosis, upper crossed postural syndrome, and scalenus anticus syndrome causing intermittent hand paresthesia. Treatment options and goals were discussed with the patient and agreed upon. These included manual therapies to improve joint, fascial, muscle, and tendon pain and restriction; activation and strengthening of inhibited muscles; improving motor control and endurance of the deep neck flexors (DNFs); improving scapular stabilization; and correction of upper crossed syndrome.

Initial treatment was provided $2 \times$ to $3 \times$ per week during the first 4 weeks. Hot moist packs, myofascial release, and postisometric relaxation were applied to the affected sternocleidomastoids, scalenes, suboccipital muscles, upper trapezii, levator scapulae, and pectorals. Diversified joint manipulation to spinal restrictions also was provided. The patient was instructed in proper biomechanics, including hip hinge and abdominal brace to support the trunk and reduce neck strain. She was advised not to lift and instructed to maintain a "packed" neck and shoulder position; slight chin retraction with shoulder depression to brace and protect her neck. ${ }^{9}$ The patient was encouraged to move in her pain-free range in an attempt to reduce fearavoidance behavior. ${ }^{10}$

Because the patient reported decreasing NP and less frequent dysphagia by week 3 , an attempt was made to assess the DNFs according to Jull et al. ${ }^{11}$ This could not be completed owing to an increase in NP during the test.

Before the start of more active rehabilitation, the patient completed the physical activity readiness questionnaire. ${ }^{12}$ During week 4, the patient reported a further decrease in pain level. The NPRS at this point was 5 to 6 of 10, which describes moderate pain and a difference from the initial score of 8 of 10 . The patient was taught the cat-camel exercise as a warm-up and to stimulate motion in her thoracic spine. Pectoral muscle stretches in the doorway were also part of her home exercise program.

In addition to rehabilitative exercise instruction, manipulation and manual myofascial treatments were provided as per findings $2 \times$ per week. Because the DNFs are stabilizers of the cervical spine and respond to inflammation by becoming weak or inhibited, the next goal was activation of the DNF in a minimal range of motion to avoid excessive 


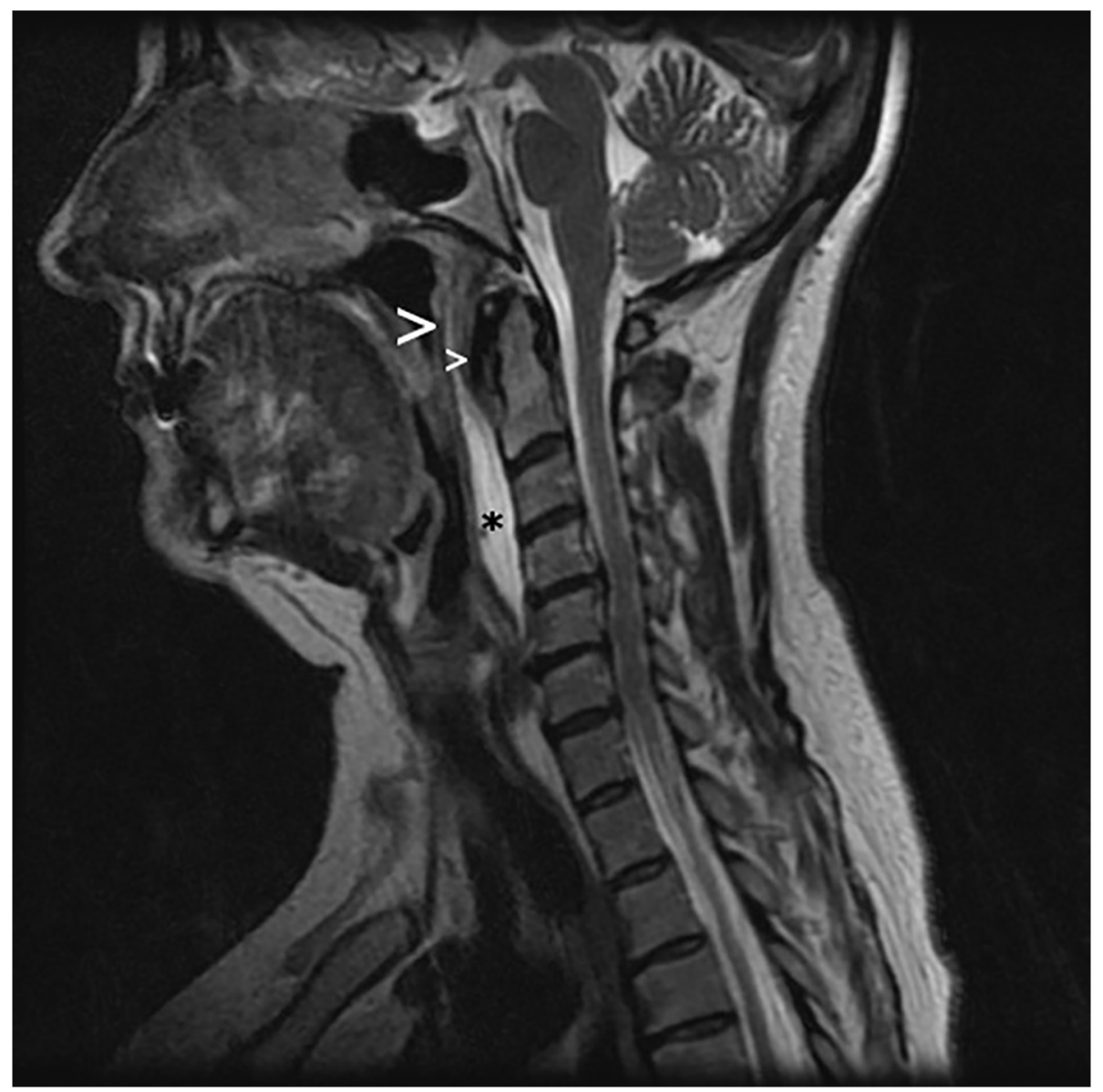

Fig 3. Parasagittal T2-weighted magnetic resonance imaging of the cervical spine demonstrates mild thickening of the retropharyngeal soft tissues (large arrowhead). Linear low signal inferior to the anterior tubercle of C1 demonstrates thickened longus colli tendon (small arrowhead). Calcification within the tendon is not appreciated in the magnetic resonance scan. Fluid accumulation/edematous changes are seen as high signal within the prevertebral soft tissues of C2-C4 (asterisk).

load on the tissues. ${ }^{13}$ Janda's neck flexion coordination test revealed the patient's chin poked forward as she raised her head off the table. This confirmed a faulty movement pattern of overactive sternocleidomastoids and inhibited DNF. During week 5, the patient was given chin retraction exercises in the sitting and standing positions while maintaining erect posture and scapulae depression. Arm abduction revealed excessive shoulder elevation on the right side before $90^{\circ}$ of abduction, which indicated an overactive upper trapezius and weak scapular stabilizers. ${ }^{13}$ Bruegger's exercises were added to activate muscles that draw the shoulders back and down and keep the neck in a neutral position, thus facilitating proper breathing. ${ }^{14}$ The patient complied with home exercises on a daily basis.

As mobility improved in the cervicothoracic spine, a scapular stabilization protocol was initiated to strengthen the lower trapezii and serratus anterior muscles. Pushup plus exercises were given while standing and facing the wall. ${ }^{15}$ Additional exercises were given to increase the strength of the lower trapezii and other scapular stabilizers. ${ }^{16}$ The patient improved considerably with chiropractic treatment and rehabilitation since the onset of the chief complaint and discharge from the hospital, and by week 8 she was discharged from care with good recovery, reporting that she only continued to have occasional mild NP, 1 to 2 of 10 on the NPRS, compared to the initial report score of 8 of 10 and a final NDI score of 6 (mild disability) compared to 30 initially. This reflects an average difference of 6.5 points on the NPRS scale and $81 \%$ improvement. The initial NDI score was 30 (severe disability), dropping to 6 on the final NDI. Initially, the patient was unable to perform Jull's test because of pain. At week 8 she held the position for 5 seconds at $26 \mathrm{mmHg}$, and maintained $28 \mathrm{mmHg}$ for 10 seconds, which indicated improved activation and strength of the DNFs. ${ }^{8}$ The patient agreed that she reached a satisfactory level of improvement, especially in view of complicating factors including degenerative spinal conditions, fibromyalgia, deconditioned state, and reliance on medication. The patient provided written permission to allow her case to be published. 


\section{DISCUSSION}

Synonyms for RCT are prevertebral calcific tendonitis and longus colli tendon hydroxyapatite deposition disease. ${ }^{17}$ Bogduk stated that RCT is a misnomer because it involves more than just the tendons; there can be inflammation and edema of the longus colli muscle from $\mathrm{C} 1$ to $\mathrm{C} 6 .{ }^{1}$ Horowitz et al. estimated an age-matched incidence of 1.31 per 100000 person-years. ${ }^{18}$ Those most susceptible to this syndrome are adults from 21 to 65 years of age with a mean age of 42 , affecting men and women equally. ${ }^{19}$

The usual presentation of RCT is one of acute, severe NP, or moderate pain that increased and became severe over a few days. The NP sometimes radiates to the occiput or shoulder and can be aggravated by swallowing and head or neck motion. There is often marked restriction of motion in the cervical spine in all directions, and palpatory tenderness can be found over the anterior, lateral, or posterior cervical spine. ${ }^{20}$ Other symptoms include dysphagia, odynophagia, and sore throat. Some patients experience mild fever and slight elevation of erythrocyte sedimentation rate, C-reactive protein, and white blood cell count. ${ }^{21,22}$

The etiology of the disease is unclear, but it is thought that excessive mechanical pressure along with degenerative changes, collagen vascular disorders, or kidney failure can lead to calcium hydroxyapatite crystal deposition in tendons. ${ }^{23}$ In this case, perhaps muscle imbalances and the faulty biomechanics associated with an upper crossed syndrome, along with preexisting degenerative changes in the cervical spine and a recent MVA, may have been at least in part responsible for her RCT.

Clinical findings in RCT can mimic those of retropharyngeal space infection. ${ }^{23,24}$ Retropharyngeal calcific tendonitis sometimes is misdiagnosed as a retropharyngeal abscess and consequently treated improperly. ${ }^{21}$ Many cases have been misdiagnosed based on imaging studies that failed to clearly indicate the lesion. ${ }^{24}$ Therefore, it is crucial to differentiate RCT from a more serious condition such as retropharyngeal abscess. ${ }^{25}$ In this case, the emergency department physicians first ordered chest films and laboratory evaluation owing to suspected infection.

No cervical spine plain films were ordered by the doctor of chiropractic or medical physicians. At the initial visit, the chiropractor recommended waiting 24 hours before obtaining plain film imaging of the patient's cervical spine. The patient agreed that if her symptoms worsened, she would contact the chiropractor immediately. Because the patient was afebrile and had no history of cancer, there was little suspicion of infection or other pathology. Also, the patient believed the neck pain resulted from lifting a toddler for whom she was a nanny. The moderate neck pain that followed an MVA 6 months prior had resolved without treatment. In retrospect, intermittent dysphagia and odynophagia should have been considered a symptom of a potentially serious condition.
The chiropractor should have ordered plain film radiographs of the patient's cervical spine to rule out pathology and the films may have demonstrated the calcific deposits.

Computed tomography is considered the gold standard for diagnosing RCT. ${ }^{21}$ Diffuse calcific deposition of early RCT may not be visible on plain film imaging, but would be more likely discernible by CT. ${ }^{26,27}$ Computed tomography may reveal discrete effusion in the retropharyngeal space versus more diffuse edema seen in abscess. However, in the early stage of abscess edema may be discrete, which can lead to a misdiagnosis of infection, making diagnosis challenging. Amorphous calcium hydroxyapatite deposits along the longus colli tendon, usually at the level of C1-C2, can be visualized on the CT with contrast. These are considered nearly pathognomonic for this condition. ${ }^{28}$ After the acute phase, CT may demonstrate resorption of calcifications. ${ }^{29}$

In this case, imaging studies revealed amorphous calcifications in the longus colli tendon and fluid collection in the prevertebral soft tissues, which led physicians to arrive at the proper diagnosis of RCT. However, they did initiate treatment for an infection and prescribed the antibacterial drug clindamycin, along with analgesics (oxycodone) and nonsteroidal anti-inflammatory drugs (NSAIDs). The patient saw her primary care provider a couple of days after discharge from the hospital, at which time he prescribed prednisone. Corticosteroids are necessary if the symptoms are severe, but in some cases successful treatment can be achieved with NSAIDs and analgesics. Reports of several cases indicate that NSAIDs, such as naproxen sodium, failed to improve the condition and patients returned to hospital because of continued pain. ${ }^{23}$

Retropharyngeal calcific tendonitis is typically a benign, self-limiting condition ${ }^{5}$ that usually resolves within 2 weeks. Debilitating pain is of great concern to the patient. Severe edema potentially can cause airway obstruction. Acute unexplained dysphagia and severe NP should be considered red flags that necessitate immediate attention to determine cause. Acute pain and inflammation typically are treated with methylprednisolone, dexamethasone, and analgesics. $^{30}$

\section{Limitations}

This is a report of 1 case. The information herein should not necessarily be extrapolated to other patients.

\section{CONCLUSIONS}

Retropharyngeal calcific tendonitis should be considered in patients seeking chiropractic care for severe neck pain associated with dysphagia and odynophagia. It is crucial that practitioners become familiar with this 
entity and recognize the clinical presentation. Clinicians considering RCT as a differential diagnosis should order or refer for the appropriate imaging, namely CT with contrast. The patient also should be provided medical management for the inflammation and pain associated with this condition. Proper initial management of the disorder in the acute stage may reduce unnecessary pain and worry for the patient. Follow-up chiropractic treatment for underlying and associated spinal and muscular dysfunctions provided durable relief in this case after the acute phase.

\section{Funding SOURCES AND Conflicts of INTEREST}

No funding sources or conflicts of interest were reported for this study.

\section{CONTRIBUTORSHIP INFORMATION}

Concept development (provided idea for the research): L.M., M.F.F., T.A.P.

Design (planned the methods to generate the results): L.M., M.F.F., T.A.P.

Supervision (provided oversight, responsible for organization and implementation, writing of the manuscript): L.M., M.F.F., T.A.P.

Data collection/processing (responsible for experiments, patient management, organization, or reporting data): L.M., M.F.F., T.A.P.

Analysis/interpretation (responsible for statistical analysis, evaluation, and presentation of the results): L.M., M.F.F., T.A.P.

Literature search (performed the literature search): L.M., M.F.F., T.A.P.

Writing (responsible for writing a substantive part of the manuscript): L.M., M.F.F., T.A.P.

Critical review (revised manuscript for intellectual content, this does not relate to spelling and grammar checking): L.M., M.F.F., T.A.P.

Other (MRI and CT image interpretation and captions): T.A.P.

\section{REFERENCES}

1. Bogduk N. The anatomy and pathophysiology of neck pain. Phys Med Rehabil Clin N Am. 2011;22(3):367-382.

2. Murphy DR. Clinical Reasoning in Spine Pain Vol. II: Primary Management of Cervical Disorders Using the CRISP Protocols. Cranston, RI: CRISP Education and Research, LLC; 2016:37.

3. Ring D, Vaccaro AR, Scuderi G, et al. Acute calcific retropharyngeal tendinitis. Clinical presentation and pathological characterization. J Bone Joint Surg Am. 1994;76(11): 1636-1642.

\section{Practical Applications}

- This report highlights an uncommon cause of NP and dysphagia and the importance of recognizing the signs of $\mathrm{RCT}$, the need to order CT imaging to make the diagnosis, and the appropriate medical care for acute inflammation and pain.

- In this case, after some improvement with medical management, the patient continued to experience considerable NP and limited mobility.

- She returned for chiropractic care and had a good recovery.

4. Trendel D, Bonfort G, Lapierre-Combes M, Salf E, Barberot J-P. Acute severe neck pain and dysphagia following a cervical maneuver: diagnostic approach. Eur Ann Otorhinolaryngol Head Neck Dis. 2014;131(1):135-138.

5. Tamm A, Jeffrey CC, Ansari K, Naik S. Acute prevertebral calcific tendinitis. J Radiol Case Rep. 2015;9(11):1-5.

6. Vernon H, Mior S. The Neck Disability Index: a study of reliability and validity. J Manipulative Physiol Ther. 1991;14 (7):409-415

7. Yeomans SG. The Clinical Application of Outcomes Assessment. Stamford, CT: Appleton \& Lange; 2000:65.

8. Liebenson C. Rehabilitation of the Spine; a Practitioner's Manual. Vol 2. Baltimore, MD: Lippincott Williams \& Wilkins; 2007:201-202.

9. Weingroff C. Packing in the neck. WBV power program. Available at: https://charlieweingroff.com/2010/11. Accessed December 31, 2018.

10. Gatchel RJ, Neblett R, Kishino N, Ray CT. Fear-avoidance beliefs and chronic pain. J Orthop Sports Phys Ther. 2016;46 (2):38-43.

11. Jull GA, O'Leary SP, Falla DL. Clinical assessment of the deep cervical flexor muscles: the craniocervical flexion test. $J$ Manipulative Physiol Ther. 2008;31(7):525-533.

12. Pescatello LS, ed. ACSM's Guidelines for Exercise Testing and Prescription. 9th ed. American College of Sports Medicine. Baltimore, MD: Lippincott Williams \& Wilkins; 2009:24.

13. Liebenson C. Functional reactivation for neck pain patients. $J$ Bodyw Mov Ther. 2002;6(1):59-66.

14. Kim K, Byun M, Lee W, Cynn H, Kwon O, Yi C. Effects of breathing maneuver and sitting posture on muscle activity in inspiratory accessory muscles in patients with chronic obstructive pulmonary disease. Multidiscip Respir Med. 2012;7(1):9.

15. Cho S-H, Baek I-H, Cheon JY, Cho MJ, Choi MY, Jung DH. Effect of the push-up plus (PUP) exercise at different shoulder rotation angles on shoulder muscle activities. J Phys Ther Sci. 2014;26(11):1737-1740.

16. Wang C-H, McClure P, Pratt NE, Nobilini R. Stretching and strengthening exercises: their effect on three-dimensional scapular kinematics. Arch Phys Med Rehabil. 1999;80(8): 923-929.

17. Eastwood J, Hudgins P, Malone D. Retropharyngeal effusion in acute calcific prevertebral tendinitis: diagnosis with CT and MR imaging. Am J Neuroradiol. 1998;19(9):1789-1792.

18. Horowitz G, Ben-Are O, Brenner A, Fliss DM, Wasserzub O. Incidence of retropharyngeal calcific tendinitis (longus colli 
tendinitis) in the general population. Otolaryngol Head Neck Surg. 2013;148(6):955-958.

19. Park R, Halpert DE, Baer A, Kunar D, Holt PA. Retropharyngeal calcific tendinitis: case report and review of the literature. Semin Arthritis Rheum. 2009;39(6):504-509.

20. Dargham HA, Bytyci F, Shuman C, Stolear A. A rare cause of acute dysphagia: acute calcific tendonitis of the longus colli muscle. BMJ Case Rep. 2017 May 27;2017.

21. Zibis AH, Giannis D, Malizos KN, Kitsioulis P, Arvanitis DL. Acute calcific tendinitis of the longus colli muscle: case report and review of the literature. Eur Spine J. 2013;22(Suppl 3): S434-S438.

22. Coulier B, Macsim M, Desgain O. Retropharyngeal calcific tendinitis-longus colli tendinitis-an unusual cause of acute dysphagia. Emerg Radiol. 2011;18(5):449-451.

23. Gabra N, Belair M, Ayad T. Case report: retropharyngeal calcific tendinitis mimicking a retropharyngeal phlegmon. Case Rep Otolaryngol. 2013.

24. Oh JY, Lim JH, Kim YS, Kwon YE, Yu JY, Lee JH. Misconceived retropharyngeal calcific tendinitis during man- agement of myofascial neck pain syndrome. Korean J Pain 2016;29(1):48-52.

25. Estimable K, Rizk C, Pujalte GG. A rare case of neck pain; acute longus colli calcific tendinitis in a possible immunocompromised individual. J Am Board Fam Pract. 2015;28(1): 146-150.

26. Offiah CE, Hall E. Acute calcific tendinitis of the longus colli muscle: spectrum of $\mathrm{CT}$ appearances and anatomical correlation. Br J Radiol. 2009;82(978):117-121.

27. Mihmanli I, Karaarslan E, Kanberoglu K. Inflammation of vertebral bone associated with acute calcific tendinitis of the longus colli muscle. Neuroradiology. 2001;43(12): 1098-1101.

28. Wasserman E, Richman A, Erdag N, Weiss R. Acute calcific prevertebral tendonitis of the longus colli muscle. Appl Radiol. 2009;38(5):31-32.

29. Haun CL. Retropharyngeal tendinitis. Am J Roentgenol. 1978;130(6):1137-1140.

30. Razon RV. Retropharyngeal calcific tendonitis: report of two cases. J Am Board Fam Med. 2009;22(1):84-88. 